\title{
Facteurs environnementaux dans l'étiologie de la maladie de Parkinson
}

\author{
J. Zayed, S. Ducic, G. Campanella, J.C. Panisset, P. André, H. Masson and M. Roy
}

\begin{abstract}
RÉSUMÉ: Nous avons examiné le rôle de l'environnement dans le développement de la maladie de Parkinson (MP). Un groupe de 42 parkinsoniens a été comparé à un groupe de 84 témoins appariés, dans le cadre d'une étude épidémiologique (1987-1989) menée sur le territoire du Département de Santé Communautaire de Valleyfield, au sud du Québec (Canada). Les rapports de cotes ajustés pour l'âge et le sexe ont été calculés pour sept facteurs environnementaux. La résidence à la campagne (OR: 0,$31 ; \mathrm{p} \leq 0,05)$ et la résidence près de mines et d'industries $(\mathrm{OR}: 0,15 ; \mathrm{p} \leq 0,05)$ sont associés à une diminution du risque de développer la MP. L'exposition professionnelle aux trois métaux Mn-Fe-Al semble être associée à une augmentation du risque de MP (OR: 2,28; $p=0,07)$ principalement lorsque la durée de l'exposition dépasse 30 ans (OR: 13,64; p $\leq 0,05)$. Les autres facteurs environnementaux qui n'ont pas été associés à la MP sont: la manipulation de pesticides, le travail à la ferme, le travail dans l'industrie et la consommation de l'eau de puits.

ABSTRACT: Environmental Factors in the Etiology of Parkinson's Disease We examined the role of the environment in the development of Parkinson's disease (PD). A group of 42 parkinsonians have been compared with a group of 84 matched controls. The epidemiological study (1987-1989) covered the territory of the Community Health Department of Valleyfield, in southern Quebec (Canada). Odds ratio adjusted for age and sex were calculated for seven environmental factors. A decreased risk for PD was associated with residence in rural areas (OR: $0.31 ; p \leq 0.05)$ and residence near industry or mining (OR: $0.15 ; \mathrm{p} \leq 0.05)$. An increased risk for PD seems to be associated with occupational exposure to the three metals $\mathrm{Mn}, \mathrm{Fe}$ and $\mathrm{Al}(\mathrm{OR}: 2.28 ; \mathrm{p}=0.07$ ) especially when the duration of exposure is longer than 30 years (OR: 13.64; $p \leq 0.05$ ). Other environmental factors not found to be associated with PD were: pesticides manipulation, farm work, industrial work and well water consumption.
\end{abstract}

Can. J. Neurol. Sci. 1990; 17:286-29l

L'apparition de la maladie de Parkinson (MP) chez les gens d'âge moyen de même que chez les gens âgés suggère un mécanisme lié au vieillissement cellulaire. En effet, l'incidence de la MP croît après le quatrième décade de viel et affecte environ $1 \%$ de la population âgée de plus de 50 ans alors qu'elle est de $0,1 \%$ pour la population dans son ensemble. ${ }^{2}$ Devant l'échec des études antécédentes pour identifier une cause à la fois nécessaire et suffisante, diverses équipes de recherche évoquent un réseau de causalité multifactorielle qui comporte à la fois une prédisposition génétique ${ }^{3}$ et une exposition à des neurotoxiques environnementaux. ${ }^{4}$

L'approche environnementale a été établie à la suite d'un incident relié à des usagers de drogues ${ }^{5.6}$ qui ont développé un syndrome parkinsonien après s'être injecté du MPTP (1-méthyl4-phényl-1,2,3,6-tétrahydropyridine). ${ }^{7}$ Cette observation, combinée au fait que le MPP+ (métabolite du MPTP) ressemble chimiquement à l'herbicide Paraquat ${ }^{8}$ et que le MPTP soit struc- turellement similaire à une variété de composés présents dans l'environnement industriel, ${ }^{9}$ a mis en évidence la possibilité que des facteurs environnementaux puissent interagir dans le développement de la MP. Cette approche, bien que considérée valable par plusieurs chercheurs, $10,11,12$ demeure controversée ${ }^{13,14}$ en raison des travaux indiquant une prévalence homogène à travers le monde, sans variation entre pays industrialisés et non industrialisés. Néamoins, l'hypothèse qu'un agent exogène puisse avoir une toxicité sélective sur le système nerveux central, produisant un syndrome parkinsonien, suscite beaucoup d'intérêt. Ainsi, plusieurs recherches suggèrent des liens entre le développement de la MP et l'exposition à des facteurs environnementaux. Ainsi, Rajput et al ${ }^{15}$ ont rapporté des agglomérats de parkinsoniens ayant consommé de l'eau de puits dans les zones rurales de la Saskatchewan. Cotzias et al ${ }^{16}$ et Emara ${ }^{17}$ ont décrit le parkinsonisme résultant d'une intoxication au manganèse. Barbeau et al18 ont trouvé une corrélation entre les

Département de médicine du travail et d'hygiène du milieu (J.Z., J.C.P.), Département de médicine sociale et préventive (S.D.), Faculté de l'éducation permanente (P.A.), Université de Montréal; Dépantement de neurologie de l'Université de Naples (G.C.); Hôpital Notre-Dame de Montréal (H.M.); Département de neurobiologie de l'Institut de Recherches Cliniques de Montréal (M.R.).

Reçu le 31 octobre 1989. Accepté le 24 avril 1990.

Tirés à part: Joseph Zayed, Ph.D., Département de médecine du travail et d'hygiène du milieu, Faculté de médecine, Université de Montréal, C.P. 6128, Succ. "A", Montréal, Québec, Canada H3C 3J7 
quantités de pesticides vendus au Québec et la distribution géographique de la MP. Enfin, Tanner et al ${ }^{19}$ ont obtenu des risques élevés quant à l'exposition à des produits chimiques industriels.

Nous avons tenté dans la présente recherche de confirmer ou d'infirmer les résultats rapportés dans la littérature quant aux facteurs de risques environnementaux, en menant une enquête sur un plan régional.

\section{MÉThOde}

Un groupe de 42 parkinsoniens et un groupe de 84 témoins appariés ont été comparés dans le cadre d'une étude épidémiologique cas-témoins amorcée en 1987 et réalisée au sud du Québec (Canada) sur le territoire du Département de Santé Communautaire (DSC) de Valleyfield (le DSC est une unité géographique et administrative de gestion des soins de santé) qui couvre 41 municipalités. Le choix de ce territoire était dicté par une population stable, une économie régionale à caractère mixte basée sur la culture maraîchère et les industries métallurgiques, et par la présence d'un bassin potentiel important de parkinsoniens, en assumant que le prévalence de la MP au Québec s'établit à 85/100 000.12

Le recensement des cas a été réalisé avec la collaboration de 76 médecins généralistes et neurologues consultants de la région sur un total de $83(91,5 \%)$. Ces derniers ont été rejoints par lettre. Nous y décrivions les grandes lignes de la recherche et faisions appel à leur collaboration pour acheminer à chacun de leurs patients parkinsoniens respectifs une enveloppe affranchie annexée à la lettre et pour nous retourner une liste comportant les initiales et la date de naissance de leurs patients. Un délai de deux mois a été accordé avant de faire un rappel téléphonique auprès de ceux qui n'avaient pas retourner cette liste. Quant aux patients, ils étaient informés du projet et étaient invités à compléter un coupon-réponse port-payé ou à téléphoner directement au chercheur principal pour signifier leur accord de participation. Pour rejoindre les patients suivis et traités l'extérieur de la région, un article a été publié à deux reprises dans les 14 hebdomadaires locaux et un numéro de téléphone de référence avait été indiqué. Pour fin de validation du diagnostic, tous les cas ont été examinés par un des deux neurologues (G.C., H.M.) collaborant à l'étude et devaient présenter 3 critères majeurs et 2 critères accessoires parmi les suivants:

\section{CRITERES MAJEURS}

1. Bradykinésie ou akinésie

2. Tremblement au repos

3. Rigidité - roue dentée, résistance plastique

4. Instabilité posturale, dos voûté, membres fléchis

5. Démarche hésitante, à petit pas

\section{CRITERES ACCESSOIRES}

1. Diminution ou absence du balancement des bras

2. Faciès figé et rareté des clignotements des yeux

3. Micrographie

4. Voix monotone et de faible volume

L'âge au début de la maladie chez les cas a été déterminé en fonction de l'année des premiers symptômes. Cette même année a aussi été appliquée aux témoins appariés pour discriminer toute exposition qui lui serait postérieure.

Les témoins ont été sélectionnés à l'aide d'un échantillonnage aléatoire simple, dans le mêmes municipalités que les cas, à partir des annuaires téléphoniques. Ils ont été appariés aux cas selon l'âge ( \pm 3 ans) et le sexe. Toutes les personnes présentant des symptômes ou des signes pouvant être compatibles avec un syndrome parkinsonien ont été excluses du groupe témoin. Tous les témoins ont été examinés par un même médecin (M.R.), possédant une longue expérience dans ce domaine.

Lors de l'examen médical à domicile des cas et des témoins, un questionnaire était complété par le médecin avec, au besoin, l'aide d'un proche parent. Dix-huit malades étaient accompagnés d'un proche parent au moment de l'entrevue: 15 conjoints, 2 fils et 1 frère. La durée de résidence commune variait de 30 à 60 ans (avec une durée moyenne de 41,3 ans). Parmi ces parkinsoniens, dix éprouvaient des difficultés d'élocution ou des pertes de mémoire et se faisaient aider. Le questionnaire, testé auprès de 5 malades et validé par 1 neurologue et 2 épidémiologistes, couvrait l'information relative aux lieux de résidence, aux contacts avec les produits chimiques agricoles, à l'historique de travail, à la consommation de tabac et de café/thé, aux chocs émotifs et physiques, aux maladies et interventions chirurgicales, au diagnostic différentiel de la MP (Shydrager, Steele Richardson, multiinfarct dementia) et aux facteurs héréditaires et familiaux.

La comparabilité générale des cas et des témoins a été calculée par le text $\chi^{220}$ pour huit variables: 1) groupe ethnique; 2) accidents de travail; 3) problèmes de santé: 4) périodes de chômage; 5) consommation de thé ou de café; 6) consommation de tabac; 7) stabilité du poids corporel et 8 ) nombre de déménagements.

De plus, les cas et les témoins ont été comparés par la méthode des rapports de cotes ("odds ratio", OR) pour sept facteurs environnementaux découlant des principales recherches 11 . 12.17 portant sur l'étiologie de la MP. Il s'agit de 1) résidence à la campagne, 2) résidence près de mines ou d'industries, 3) utilisation de pesticides, 4) travail sur la ferme, 5) travail dans l'industrie, 6) manipulation de $\mathrm{Fe}, \mathrm{Mn}$ et $\mathrm{Al}$ et 7) consommation d'eau de puits.

Pour fin d'analyse, toutes les expositions d'une durée minimale d'une année et antérieures aux premiers symptômes de la maladie ont été retenues.

Le OR estimé a été ensuite ajusté par la technique de MantelHaenszel ${ }^{21}(\mathrm{M}-\mathrm{H})$ pour contrôler les effets de l'âge el du sexe pour des séries appariées et sa signification a été calculée par $\chi^{2}$ de M-H. ${ }^{21}$ Les intervalles de confiance du OR ont subséquemment été calculés comme décrits par Miettinen. ${ }^{21}$ Les risques ont aussi été calculés en fonction de la durée d'exposition. Un test $\chi^{2}$ pour les tendances a permis de mesurer les relations dosesréponses. Enfin, les risques ont été estimés en fonction de l'année du début des symptômes (avant 1971-de 1971 à 1979-après 1979).

\section{RÉsultats}

Les médecins nous ont retourné des listes comportant 69 initiales et dates de naissance. Quatre d'entre eux étaients en duplicata et un en triplicata. Sept cas nous ont directement rejoint à la suite de la parution des articles dans les hebdomadaires.

En tout, 57 parkinsoniens distribués dans 22 municipalités ont accepté de participer à la recherche. Quarante-deux de ces derniers ont vu leur diagnostic confirmé, alors que les autres ont dû être exclus. En effet, 12 ont vu leur diagostic invalidé (il s'agissait plutôt d'artériosclérose cérébrale, de parkinsonisme 


\begin{tabular}{|c|c|c|c|c|}
\hline Variables & Sous-variables & Cas & Témoins & $\chi^{2}$ \\
\hline Groupe ethnique & $\begin{array}{l}\text { Français } \\
\text { Anglais } \\
\text { Autre }\end{array}$ & $\begin{array}{r}35 \\
5 \\
2\end{array}$ & $\begin{array}{r}75 \\
6 \\
2\end{array}$ & Non significatif \\
\hline Accidents de travail & $\begin{array}{l}\text { Oui } \\
\text { Non }\end{array}$ & $\begin{array}{l}12 \\
30\end{array}$ & $\begin{array}{l}17 \\
67\end{array}$ & Non significatif \\
\hline Problèmes de santé & $\begin{array}{l}\text { Oui } \\
\text { Non }\end{array}$ & $\begin{array}{r}3 \\
39\end{array}$ & $\begin{array}{l}10 \\
74\end{array}$ & Non significatif \\
\hline Périodes de chômage & $\begin{array}{l}\text { Oui } \\
\text { Non }\end{array}$ & $\begin{array}{r}7 \\
32\end{array}$ & $\begin{array}{l}18 \\
66\end{array}$ & Non significatif \\
\hline $\begin{array}{l}\text { Consommation de thé/café } \\
\text { (nombre de tasses) }\end{array}$ & $\begin{array}{r}0 \\
1 \\
2 \\
3 \\
\geq 4\end{array}$ & $\begin{array}{r}8 \\
6 \\
6 \\
14 \\
8\end{array}$ & $\begin{array}{r}5 \\
15 \\
16 \\
30 \\
17\end{array}$ & Non significatif \\
\hline Consommation de tabac & $\begin{array}{l}\text { Fumeur } \\
\text { Ex fumeur } \\
\text { Non fumeur }\end{array}$ & $\begin{array}{r}1 \\
23 \\
18\end{array}$ & $\begin{array}{l}20 \\
27 \\
37\end{array}$ & $\mathrm{p} \leq 0,05$ \\
\hline Perte de poids importante & $\begin{array}{l}\text { Oui } \\
\text { Non }\end{array}$ & $\begin{array}{l}18 \\
23\end{array}$ & $\begin{array}{l}34 \\
50\end{array}$ & Non significatif \\
\hline Nombre de déménagements & $\begin{array}{r}0 \\
1 \\
2 \\
3 \\
\geq 4\end{array}$ & $\begin{array}{r}5 \\
11 \\
7 \\
8 \\
11\end{array}$ & $\begin{array}{r}5 \\
20 \\
19 \\
20 \\
20\end{array}$ & Non significatif \\
\hline
\end{tabular}

Tableau 2: Tableau de Contingence pour les Triplets et Calcul du Risque (OR)

\begin{tabular}{|c|c|c|c|c|c|c|c|c|}
\hline \multirow[b]{2}{*}{ Facteurs Environnementaux } & \multicolumn{6}{|c|}{$\begin{array}{c}\text { Exposition } \\
\text { Séries Appariées }\end{array}$} & \multirow{2}{*}{$\begin{array}{c}\text { Rapport des Cotes } \\
\text { OR (M-H) } \\
\text { IC } 95 \% \text { (Miettinen) } \\
\end{array}$} & \multirow{2}{*}{$\begin{array}{c}\text { Test de } \\
\text { Signification } \\
\text { (Mantel-Haenszel }\end{array}$} \\
\hline & +++ & $\begin{array}{l}+++ \\
++\end{array}$ & +-- & -++ & $\begin{array}{l}--+ \\
-+-\end{array}$ & --- & & \\
\hline Résidence à la campagne & 29 & 4 & 0 & 5 & 3 & 1 & $\begin{array}{c}0,31 \\
(0,11-0,91)^{*}\end{array}$ & $S(p<0,05)$ \\
\hline $\begin{array}{l}\text { Résidence près de mines ou } \\
\text { d'industries }\end{array}$ & 7 & 3 & 1 & 10 & 13 & 8 & $\begin{array}{c}0,15 \\
(0,04-0,55)^{*}\end{array}$ & $S(p<0,05)$ \\
\hline Utilisation de pesticides & 9 & 12 & 2 & 2 & 9 & 8 & $\begin{array}{c}1,23 \\
(0,46-3,29)^{*}\end{array}$ & NS $(p=0,35)$ \\
\hline Travail à la ferme & 8 & 5 & 4 & 2 & 13 & 10 & $\begin{array}{c}0,65 \\
(0,25-1,69)^{*}\end{array}$ & NS $(p=0,24)$ \\
\hline Travail dans l'industrie & 0 & 0 & 5 & 1 & 3 & 33 & $\begin{array}{c}2,00 \\
(0,62-6,36)^{*}\end{array}$ & $N S(p=0,11)$ \\
\hline Manipulation de $\mathrm{Mn}, \mathrm{Fe}, \mathrm{Al}$ & 0 & 2 & 7 & 1 & 5 & 27 & $\begin{array}{c}2,28 \\
(0,85-2,77)^{*}\end{array}$ & NS $(p=0,07)$ \\
\hline $\begin{array}{l}\text { Consommation de l'eau } \\
\text { de puits }\end{array}$ & 19 & 12 & 1 & 2 & 6 & 2 & $\begin{array}{c}1,40 \\
(0,48-4,02)^{*}\end{array}$ & NS $(p=0,29)$ \\
\hline
\end{tabular}

*Les chiffres entre parenthèses indiquent les intervalles de confiance à $95 \%$.

Le premier symbole des séries appariées représente le cas et les deux autres représentent les témoins appariés. Une exposition est identifiée par un "+" et son absence par un "-". 
Tableau 3: Durée d'Exposition aux Facteurs Environnementaux et Risque de Maladie de Parkinson

\begin{tabular}{|c|c|c|c|c|c|}
\hline Facteurs Environnementaux & Durée & Cas (n) & Témoins (n) & Rapports des Cotes OR & IC $95 \%$ \\
\hline Résidence près de mines et industries* & $\begin{array}{l}\text { jamais } \\
1-10 \text { ans } \\
11-20 \text { ans } \\
21-30 \text { ans } \\
>30 \text { ans }\end{array}$ & $\begin{array}{r}31 \\
5 \\
2 \\
3 \\
1\end{array}$ & $\begin{array}{r}34 \\
33 \\
6 \\
8 \\
3\end{array}$ & $\begin{array}{l}1,00 \\
0,17 \\
0,37 \\
0,41 \\
0,37\end{array}$ & $\begin{array}{c}\overline{0} \\
0,05-0,52 \\
0,07-2,25 \\
0,07-2,78 \\
0,04-4,31\end{array}$ \\
\hline Utilisation de pesticides & $\begin{array}{l}\text { jamais } \\
1-10 \text { ans } \\
11-20 \text { ans } \\
21-30 \text { ans } \\
>30 \text { ans }\end{array}$ & $\begin{array}{r}19 \\
6 \\
4 \\
5 \\
8\end{array}$ & $\begin{array}{r}41 \\
16 \\
8 \\
10 \\
9\end{array}$ & $\begin{array}{l}1,00 \\
0,81 \\
1,08 \\
1,08 \\
1,92\end{array}$ & $\begin{array}{c}-\overline{0,24-2,68} \\
0,24-4,66 \\
0,24-4,12 \\
0,56-6,57\end{array}$ \\
\hline Travail dans l'industrie & $\begin{array}{l}\text { jamais } \\
1-10 \text { ans } \\
11-20 \text { ans } \\
21-30 \text { ans } \\
>30 \text { ans }\end{array}$ & $\begin{array}{r}37 \\
2 \\
2 \\
1 \\
0\end{array}$ & $\begin{array}{r}79 \\
3 \\
1 \\
1 \\
0\end{array}$ & $\begin{array}{l}1,00 \\
1,42 \\
4,27 \\
2,13 \\
\text { ND }\end{array}$ & $\begin{array}{c}-\overline{0,23-11,11} \\
0,45-40,70 \\
0,14-33,30 \\
-\end{array}$ \\
\hline Manipulation de $\mathrm{Mn}, \mathrm{Fe}, \mathrm{Al}{ }^{* *}$ & $\begin{array}{l}\text { jamais } \\
1-10 \text { ans } \\
11-20 \text { ans } \\
21-30 \text { ans } \\
>30 \text { ans }\end{array}$ & $\begin{array}{r}33 \\
0 \\
1 \\
2 \\
6\end{array}$ & $\begin{array}{r}75 \\
5 \\
3 \\
0 \\
1\end{array}$ & $\begin{array}{c}1,00 \\
\text { ND } \\
0,76 \\
\text { ND } \\
13,64\end{array}$ & $\begin{array}{c}\overline{-} \\
0,08-8,69 \\
\overline{-} \\
1,52-76,28\end{array}$ \\
\hline
\end{tabular}

*Test $\chi^{2}$ pour les tendances: $\chi^{2}=4,56 ; \mathrm{df}=1 ; p=0,03$

**Text $\chi^{2}$ pour les tendances: $\chi^{2}=8,92 ; \mathrm{df}=1 ; \mathrm{p}=0,003$

***ND $=$ non déterminable

médicamenteux, de syndrôme confusionnel, de tremblements essentiel, postural et sénile), 2 résidaient hors du territoire, et 1 a dû être exclu en raison du stade terminal de la maladie, d'où un diagnostic impossible. Quant aux témoins, 3 ont été exclus parce qu'ils présentaient un syndrome parkinsonien ( 2 présentaient les premiers signes de la MP et 1 présentait un parkinsonisme médicamenteux). Environ deux-tiers des parkinsoniens (27/42 ou $64,3 \%)$ sont des hommes et un tiers (15/42 ou 35,7\%) sont des femmes. L'âge moyen des hommes au moment de l'apparition des premiers symptômes se situe à $55,9\left(S_{x}=11,6\right)$ et celui des femmes à 61,1 ans $\left(S_{x}=9,1\right)$. Cet âge, pour les deux sexes $(57,8 \pm 11,1) n$ 'est pas significativement différent de celui que l'on retrouve dans la littérature. ${ }^{2}$

Au niveau de la comparabilité générale, le tableau 1 montre que seule la consommation de tabac diffère significativement ( $p=0,05$ ) entre les cas et les témoins pour les catégories "fumeur" et "ex-fumeur". La proportion du nombre de fumeurs chez les cas est nettement inférieure à celle des témoins, ce qui concorde avec les études antérieures. ${ }^{22.23}$

Parmi les facteurs environnementaux (tableau 2), la résidence à la campagne $(O R=0,31)$ et la résidence près de mines ou d'industries ( $O R=0,15$ ) constituent les seules variables dont les résultats sont significatifs $(p=0,05)$. L'utilisation de pesticides $(O R=1,23)$, le travail en industrie $(O R=2,00)$, la manipulation de $\mathrm{Mn}$, Fe et $\mathrm{Al}(\mathrm{OR}=2,28)$ et la consommation de l'eau de puits $(\mathrm{OR}=1,40$ ), malgré leur risque supérieur à 1 , montrent des résultats non significatifs. Toutefois, la manipulation de $\mathrm{Mn}, \mathrm{Fe}$ et $\mathrm{Al}$ présente un risque significatif au seuil de $0,1(p=0,07)$. De plus, comme l'indique le tableau 3, les sujets qui ont manipulé ces 3 métaux durant plus de 30 ans ont un risque significatif élevé $(\mathrm{OR}=13,64$; IC 95\%: 1,52-76,28) quoique le nombre de cas (6) et de témoins (1) amène des variations importantes. Le test khicarré pour les tendances montre une relation dose-réponse significative traduite par une augmentation du risque en fonction de 
l'augmentation de la durée d'exposition pour la résidence près de mines et d'industries $(p=0,03)$ et pour la manipulation des trois métaux $(p=0,003)$.

Enfin, l'estimation des risques en fonction de l'année du début des symptômes montre une stabilité pour l'ensemble des facteurs environnementaux sauf pour l'utilisation des pesticides et la consommation de l'eau de puits. Pour ces deux variables, les estimations des risques fluctuent respectivement à la baisse et à la hausse en fonction des années les plus récentes.

\section{Discussion}

Menée sur un plan régional, cette étude a permis d'interviewer 42 cas de MP et 84 témoins. Les résultats obtenus indiquent une association négative entre la résidence en milieu rural et la résidence près de mines et d'industries par rapport au risque de MP. Cet élément a aussi été observé par Tanner et al. ${ }^{19}$ Il peut être expliqué par la nature même d'une exposition de type environnemental aux métaux et aux pesticides; cette exposition étant moins sévère qu'une exposition de type professionnel. Ceci concorde d'ailleurs avec les résultats du deuxième volet de la présente étude où l'exposition environnementale aux métaux et aux pesticides a été mesurée et ne montre aucune différence significative entre les cas et les témoins. ${ }^{24}$ Par ailleurs, il est possible que l'exposition dans les municipalités où sont situées les industries et les mines soit moins importante que dans les municipalités qui les voisinent à cause du gradient de dispersion du panache relatif aux rejets atmosphériques. En effet, ce panache peut être représenté pour une courbe gaussienne, fonction de la hauteur des cheminées, du poids des particules et de la direction des vents dominants. ${ }^{25}$ Ainsi, le dépôt de ces rejets et l'exposition qui en découle peuvent donc se produire à une distance plus ou moins grande du lieu de leur émission.

Les associations observées entre les quatre facteurs environnementaux présentant des risques supérieurs à 1 et le développement de la MP convergent dans le même sens que d'autres recherches. ${ }^{3,11}$ Notamment, une étude cas-témoins récente et similaire à la nôtre, menée en Espagne (Madrid) a établi des associations entre la MP et l'exposition aux pesticides et la consommation de l'eau de puits. ${ }^{26}$ Tanner et al ${ }^{19}$ ont trouvé une association entre l'exposition professionnelle à des produits chimiques industriels et une augmentation du risque de développement de la MP. Dans notre étude, le facteur relatif à la manipulation de Mn$\mathrm{Fe}-\mathrm{Al}$ retient davantage l'attention à cause de sa proximité du seuil de signification à $0,05(p=0,07)$. Cette observation rejoint celle de Aquilonius et Hartvig 27 qui ont déterminé la distribution géographique de la MP en Suède et qui ont postulé la responsabilité des métaux lourds dans l'étiologie de la maladie, à cause de la forte prévalence de la MP dans un comté caractérisé par la présence exclusive d'une industrie sidérurgique et d'une papeteric. D'ailleurs le manganèse avait déjà été associé à la MP,16,17 alors que des mineurs exposés à la poussière de ce métal avaient développé un syndrome parkinsonien. Poirier et al ${ }^{28}$ ont relié l'exposition au fer à une augmentation des radicaux libres et au développement subséquent de la MP. Quant à l'aluminium, Yanagihara et al ${ }^{29}$ et Piccardo et al ${ }^{30}$ l'ont relié à la demence parkinsonienne en Guam.

De tous les facteurs environnementaux, deux présentaient des fluctuations temporelles des risques en fonction de l'année du début des symptômes. L'interprétation de la diminution du risque pour l'utilisation des pesticides, pourrait résider dans la nature moins toxique des pesticides utilisés présentement, en dépit de leurs quantités accrues. Quant à l'augmentation du risque en fonction de la consommation de l'eau de puits, elle peut être expliquée par le fait que cette eau continue de concentrer des résidus toxiques infiltrés et migrant dans le sol, peu importe leur origine temporelle et hormis le fait que l'utilisation de plusieurs de ces contaminants soit maintenant interdite.

Enfin, malgré le manque de signification statistique pour plusieurs facteurs environnementaux étudiés, dû possiblement, à la taille de l'échantillon, les résultats de cette recherche menée sur un plan régional semblent converger avec les résultats des récentes études sur la MP. 19,22,26

\section{REMERCIEMENTS}

Ce travail a été réalisé avec la collaboration du Département de Santé Communautaire de Valleyfield, Québec, Canada. Nous désirons remercier Dr Antoine Simard pour la révision du manuscrit et Mme Danielle Gaudreau pour son support technique.

\section{RÉFÉRENCES}

1. Rajput AH, Offord KP, Beard MC, et al. Epidemiology of parkinsonism: incidence, classification, and mortality. Ann Neurol 1984; 16: 278-282.

2. Rondot P, Recondo J. La maladie de parkinson. Paris: J.B. Baillière 1976.

3. Barbeau A. Etiology of Parkinson's disease: a research strategy. Can J Neurol Sci 1984; 11: 24-28.

4. Calne DB, Langston JW. Aetiology of Parkinson's disease. Lancet 1983, ii: 1457-1459.

5. Langston JW, Ballard P, Tetrud JW, et al. Chronic parkinsonism in humans due to a product of meperidine-analog synthesis. Science 1982; 219: 979-980.

6. Lewin R. Trail of ironies to Parkinson's disease. Science 1984; 224: 1083-1085.

7. Irwin I, Langston JW. Selective accumulation of MPP+ in the substantia nigra: a key to neurotoxicity? Life Sci 1985; 36: 207 212.

8. Barbeau A, Dallaire L, Buu NT, et al. New amphibian models for the study of 1-methyl-4-phenil-1,2,3,6-tetrahydropiridine (MPTP). Life Sci 1985; 37: 1125-1134.

9. Solomon H, D'Amato RJ. Predicting Parkinson's disease. Nature 1985; 317: 198-199.

10. Mena I, Marin O, Fuenzalida A. Chronic manganese poisoning: clinical picture and manganese turnover. Neurology 1976; 17: 128-136.

11. Rajput AJ, Stern W, Christ A. Etiology of Parkinson's disease: environmental factor(s). Neurology 1984; 34 (Suppl 1): 207.

12. Barbeau A, Roy M, Bernier G, et al. Ecogenetics of Parkinson's disease: prevalence and environmental aspects in rural areas. Can J Neurol Sci 1987; 14: 36-41.

13. Eldridge R, Rocca WA. The clinical syndrome of striatal dopamine deficiency: parkinsonism induced by MPTP. New Engl J Med 1985; 313: 1159-1160.

14. Marttila RJ, Rinne UK. Epidemiology of Parkinson's disease an overview. J Neurol Transm 1981; 51: 135-148.

15. Rajput AH, Uitti RJ, Stern W, et al. Early onset of Parkinson's disease in Saskatchewan - environmental considerations for etiology. Can J Neurol Sci 1986; 13: 312-316.

16. Cotzias GC, Papavisiliou PS, Ginos J, et al. Metabolic modification of Parkinson's disease and of chronic manganese poisoning. Ann Rev Med 1971; 22: 305-326.

17. Emara AM. Chronic manganese poisoning in the dry battery industry. Brit J Indust Med 1971; 28: 78-84.

18. Barbeau A, Roy M, Cloutier L, et al. Environmental and genetic factors in the etiology of Parkinson's disease. Adv Neurol 1986; 45: $299-306$ 
19. Tanner CM, Chen B, Wang W-Z, et al. Environmental factors and Parkinson's disease: a case-control study in China. Neurol 1989; 39: 660-664.

20. Siegel S. Nonparametric statistics for the behavioral sciences. New York: McGraw-Hill, 1956.

21. Kahn HA. An introduction to epidemiologic methods. New York: Oxford University Press, 1983.

22. Ho SC, Woo J, Lee CM. Epidemiologic study of Parkinson's disease in Hong Kong. Neurol 1989; 39: 1314-1318.

23. Godwin-Austen RB, Lee PN, Marmot MG, et al. Smoking and Parkinson's disease. J Neurol Neurosurg Psychiat 1982; 45: 577581.

24. Zayed J, André P, Panisset JC, et al. Environmental contamination by metals and Parkinson's disease. Water, Air and Soil Pol 1990; 49: 197-203.

25. Jorgensen SE, Johnsen I. Principals of environmental science and technology. New York: Elsevier 1989.

26. Jimenez-Jimenez FJ, Gonzales DM, Gimenez-Roldan S. Exposure to well water drinking and pesticides in Parkinson's disease: a case-control study from the southeast area of Madrid. Proceedings of the Ninth International Symposium on Parkinson's disease, World Congress of Neurology 1988; 118.

27. Aquilonius SM, Hartvig P. Utilization of antiparkinson drugs in Sweden. Upsala J Med Sci 1986; (Suppl 43): 93.

28. Poirier J, Dallaire L, Barbeau A. Parkinson's disease and free radicals. Free radicals, cell damage and disease. London: Catherine Rice-Evans, 1986.

29. Yanagihara R, Garruto RM, Gajdusek DC, et al. Calcium and vitamin D metabolism in Guamania chamorros with amyotrophic lateral sclerosis and parkinsonism-dementia. Ann Neurol $1984 ; 15 ; 42-48$.

30. Piccardo P, Yanagihara R, Garruto RM, et al. Histochemical and $\mathrm{X}$-ray microanalytical localization of aluminium in amyotrophic lateral sclerosis and parkinsonism-dementia of Guam. Acta Neuropathol $1988 ; 71: 1-4$. 